\title{
Foot Drop
}

National Institute of Neurological Disorders and Stroke (NINDS)

\section{Source}

National Institute of Neurological Disorders and Stroke (NINDS). Foot Drop Information

Page.

Foot drop describes the inability to raise the front part of the foot due to weakness or paralysis of the muscles that lift the foot. As a result, individuals with foot drop scuff their toes along the ground or bend their knees to lift their foot higher than usual to avoid the scuffing, which causes what is called a "steppage" gait. Foot drop can be unilateral (affecting one foot) or bilateral (affecting both feet). Foot drop is a symptom of an underlying problem and is either temporary or permanent, depending on the cause. Causes include: neurodegenerative disorders of the brain that cause muscular problems, such as multiple sclerosis, stroke, and cerebral palsy; motor neuron disorders such as polio, some forms of spinal muscular atrophy and amyotrophic lateral sclerosis (commonly known as Lou Gehrig's disease); injury to the nerve roots, such as in spinal stenosis; peripheral nerve disorders such as Charcot-Marie-T ooth disease or acquired peripheral neuropathy; local compression or damage to the peroneal nerve as it passes across the fibular bone below the knee; and muscle disorders, such as muscular dystrophy or myositis. 